\title{
Personal Authentication Based on Wrist EMG Analysis by a Convolutional Neural Network
}

\author{
Ryohei Shioji*, Shin-ichi Ito*, Momoyo Ito ${ }^{+}$and Minoru Fukumi ${ }^{+}$ \\ Tokushima University, \\ 2-1, Minami-Josanjima, Tokushima, 770-8506, Japan \\ *\{c501737029, s.ito $\} @$ tokushima-u.ac.jp \\ +\{momoito, fukumi\}@is.tokushima-u.ac.jp
}

\begin{abstract}
Recent years, biological signals have attracted much attention as a tool of human interface. Electromyogram $(E M G)$ has been used in a variety of situations in particular. We measure EMG of arms or shoulders in many cases. In addition, we often use expensive wet type sensors. However, they are inconvenient and high-cost. On the one hand, there have been few works of personal authentication using EMG. Therefore, in this paper we measure EMG by attaching dry type sensors to wrist, and carry out personal authentication. The conventional method in this paper is divided into three units such as a measuring, a feature extraction, and a discrimination units. We measure EMG signals with eight dry type sensors on the wrist. After that, we identify a motion opening our hands. We use a convolutional neural network (CNN) to learning and authentication. We collected 40 data for each subject. The average accuracy of two-class separation was $94.9 \%$ by CNN. In addition to the conventional method, the proposed method in this paper preprocesses the data. Large noise was removed using a high path filter. By this preprocessing, identification accuracy (Two-class classification using $\mathrm{CNN}$ ) improved by $1.5 \%$. The true acceptance rate improved by $7.2 \%$, and the false acceptance rate improved by $0.0067 \%$.
\end{abstract}

Keywords: Biometrics, CNN, Deep learning, Wrist EMG, Personal authentication.

\section{Introduction}

Recent years, biological signals have attracted attention as a tool of human interface. Biological signals such as brain waves, the pulse wave and electromyogram
(EMG) have been actively researched. Above all, EMG has been applied to various researches to date. For example, it has been used as a control signal of an electric prosthetic hand. Furthermore, there have been pointing devices [1] and eating support systems using EMG [2].

In many of conventional researches [3], EMG signals were measured by attaching sensors to an arm or a shoulder, because there are many muscle mass. However, it causes inconvenience in attachment and detachment of sensors in everyday life. In addition, most of sensors measuring EMG were wet type ones. They can measure EMG noiselessly. However, there is an additional cost for disposable electrodes. Because of these reasons, techniques using EMG has not spread to our daily lives.

In addition, there have been several researches of behavior authentication using EMG. However the researches of personal authentication is less.

From these backgrounds, in this paper we measure EMG by attaching dry type sensors to wrist, and carry out personal authentication based on EMG. The ultimate goal is to achieve online personal authentication. As the first step, we carry out offline personal authentication in this paper. First, we collect motion data spreading our hands. After that, we carry out personal authentication for the motion.

\section{Related work}

There has been much work using various biological signals for personal authentication. For example, Electroencephalogram (EEG), Electrooculography (EOG), Mechanomyogram (MMG), Galvanic skin response (GSR), Electrocardiogram (ECG) and Electromyogram (EMG) [4]. The reference [5] achieved $99.8 \%$ accuracy in personal 


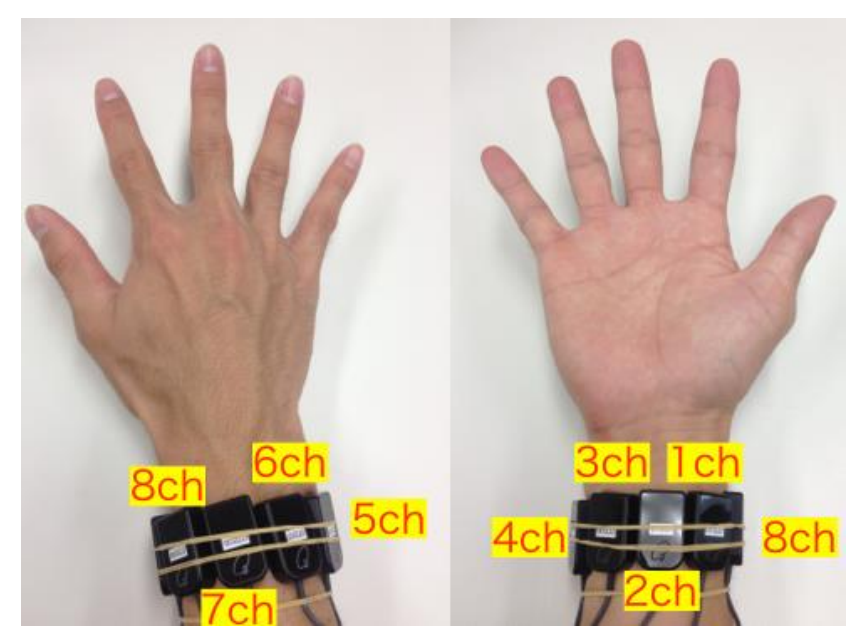

Fig. 1. Sensor Position of the Proposed Method.

authentication using Electroencephalogram that occurred in eye movement. Individual authentication using electrocardiogram achieved $100 \%$ accuracy [6]. However, measurement device is large-scale. In addition, personal authentication using IRIS [7] was presented, which gave accuracy rate of $100 \%$. However, its accuracy decreased to $76.67 \%$ in a case that contact lenses influenced IRIS images. On the one hand, average accuracy was $90 \%$ in personal authentication using EMG [8]. However, it is cumbersome and inconvenient mounting a device in the forearm. Incidentally, the average accuracy rate of a motion opening our hand is $93.75 \%$ [9]. Motion identification accuracy with wrist EMG was 94.8\% [3].

Generally, false acceptance rate (FAR) in personal authentication must be zero $\%$ or near zero $\%$. Therefore we try to reduce FAR by a preprocessing in this paper. In the next section, we describe a conventional method. [9]

\section{Conventional method}

In this section, we describe the previous implementation of our paper [9]. The conventional method is divided into three units such as a measuring, a feature extraction and a discrimination units.

\subsection{Measuring}

The measuring unit of the conventional method measures EMG by 8 channels of dry type sensors. We attach sensors around a wrist as Fig.1 shows. There is a reason to use 8 channels. If a wrist EMG is fine at a subject, individual features can appear in it. The EMG relating to a hand motion can include information of corresponding extensor muscle. In addition, we try to prevent positional

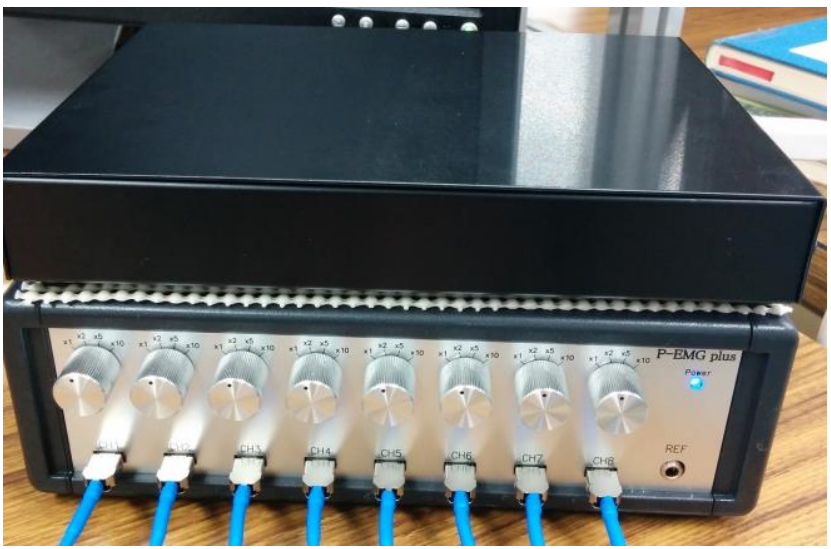

Fig. 2. P-EMG plus.

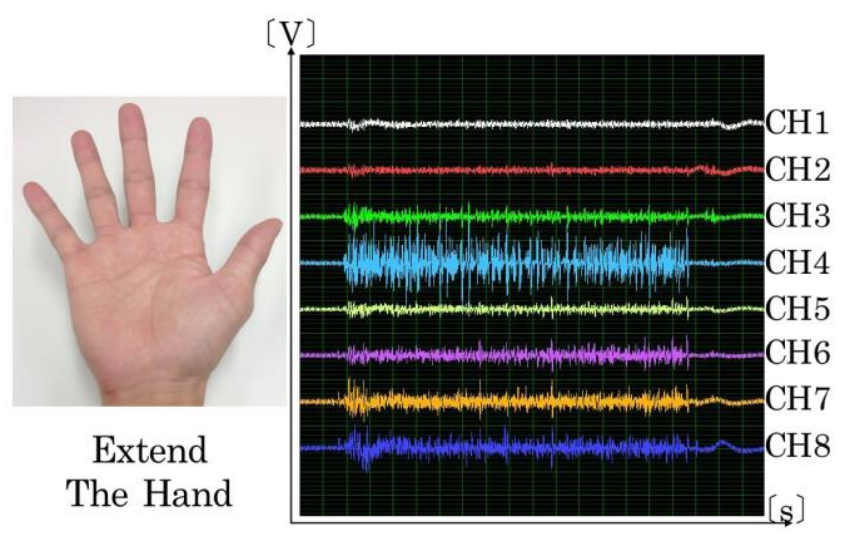

Fig. 3. Samples of EMG Data.

deviation.

We use P-EMG plus (Fig.2) for measuring EMG [1]. Sampling rate in measuring EMG is $1 \mathrm{kHz}$. We carry out personal authentication using EMG by the motion extending our hands. Samples of EMG data are shown in Fig.3.

\subsection{Feature extraction}

In the feature extraction, we use a Convolutional Neural Network (CNN). Fig.4 shows its detailed layer structure. The output of $\mathrm{CNN}$ leads to a discrimination unit. Details of the discrimination unit are described in the next subsection. The configuration of the layers is simple. We obtain $128 \times 8$ input data from the measuring unit. We extract adjacent inter-channel features. Then, a filter size of the convolution layers is $3 \times 3$. CNN does not contain pooling layers in this paper. It is unlikely to cause positional deviation because the same wavelengths continue in EMG signals.

\subsection{Discrimination}

In the discrimination, we use full connection layers. 


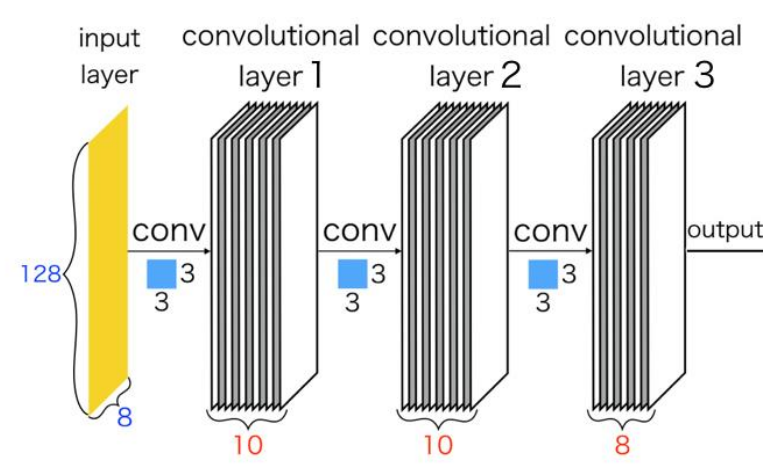

Fig. 4. Layer Structure of the CNN followed by full connection layer.

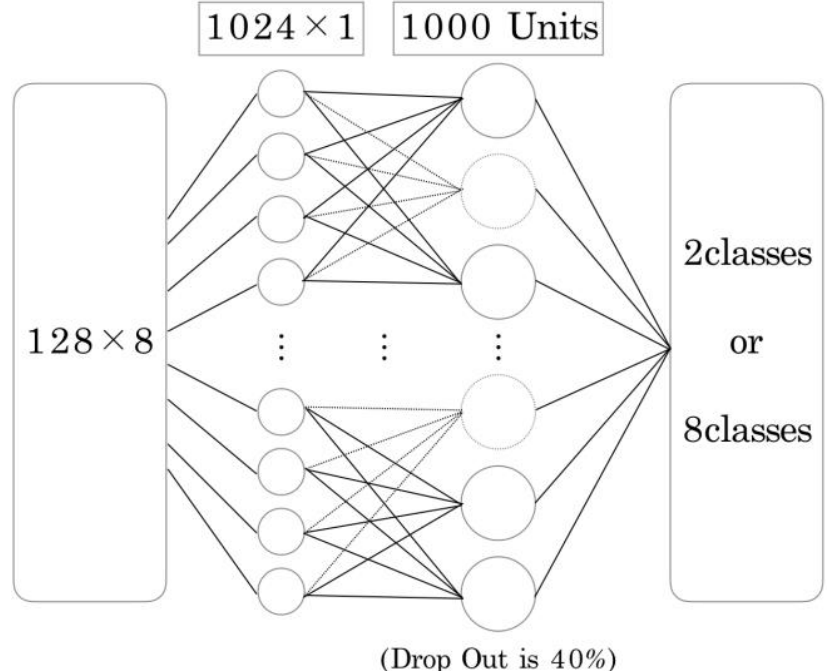

Fig. 5. Layer Structure of full connection layer.

Fig.5 shows the configuration of full connection layers. In addition, we try to prevent overfitting by using drop-out in the full connection layer. The rate of drop-out is $40 \%$. The number of output units is the same as that of classes in pattern classification. The first pattern classification is whether a hand motion belongs to a particular one of all subjects. This is two-class classification. The second pattern classification is whether a hand motion is whose operation. This is multi-class classification. In the two-class classification, the number of output units is 2 . One of two output units corresponds to the particular class and the other output unit corresponds to the other class. In the multi-class classification, the number of output units is the same as that of subjects. Each output unit in this case corresponds to each class. The structure of the output layer is the same even in CNN.

\section{Proposed method}
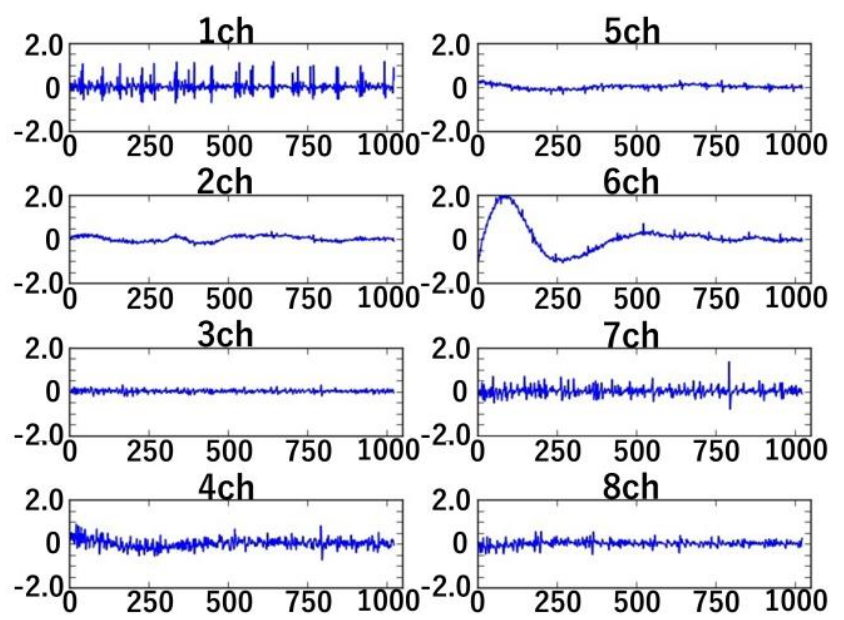

Fig. 6. Unprocessed Measurement Data.
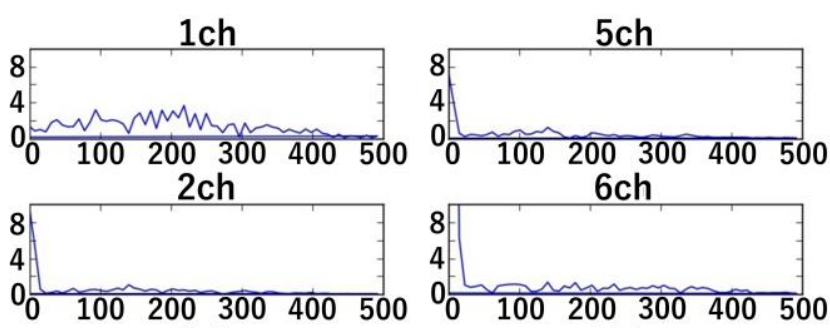

3 ch
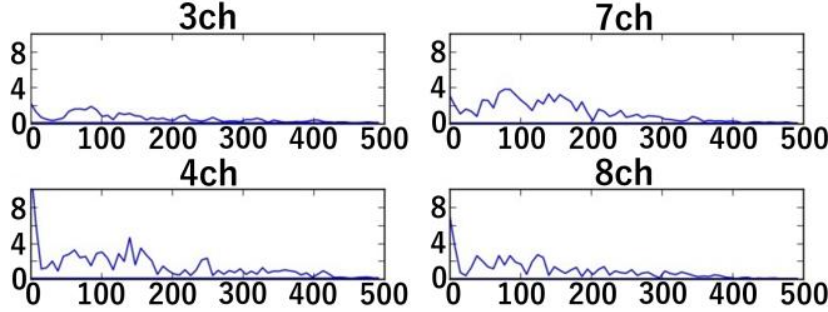

Fig. 7. Power Spectrum after FFT.

In the conventional method, the measurement data is not preprocessed. On the one hand, in the proposed method, we preprocess measurement data. Fig.6 shows measurement data contain large drift noises. Therefore, we perform FFT on measurement data and perform frequency analysis. Fig.7 shows the power spectra after FFT. We can see that frequencies below $20 \mathrm{~Hz}$ significantly have large values. Therefore, we performed a high pass filter. Fig.8 shows the power spectra after performing a high pass filter. Thereafter, we perform inverse FFT and obtain input data. Fig.9 shows measurement data after pre-processing.

\section{Experiment}

The subjects of experiments are 8 people. The subjects repeated the hand motion and faintness 10 times about every three seconds. This is 1 trial of EMG measurement. Fig.10 shows a measurement flow of EMG. We conducted this measurement 4 days on each subject. Therefore, we 
$1 \mathrm{ch}$
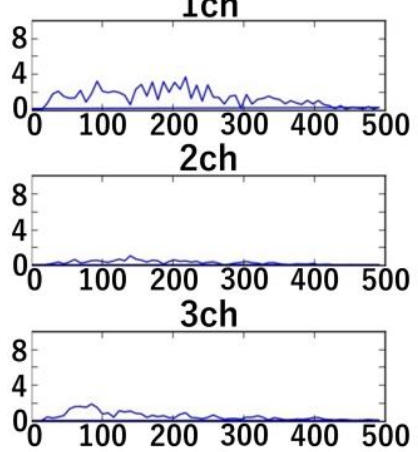

$4 \mathrm{ch}$

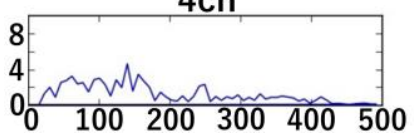

Fig. 8. Power Spectrum after FFT \& High Path Filter.
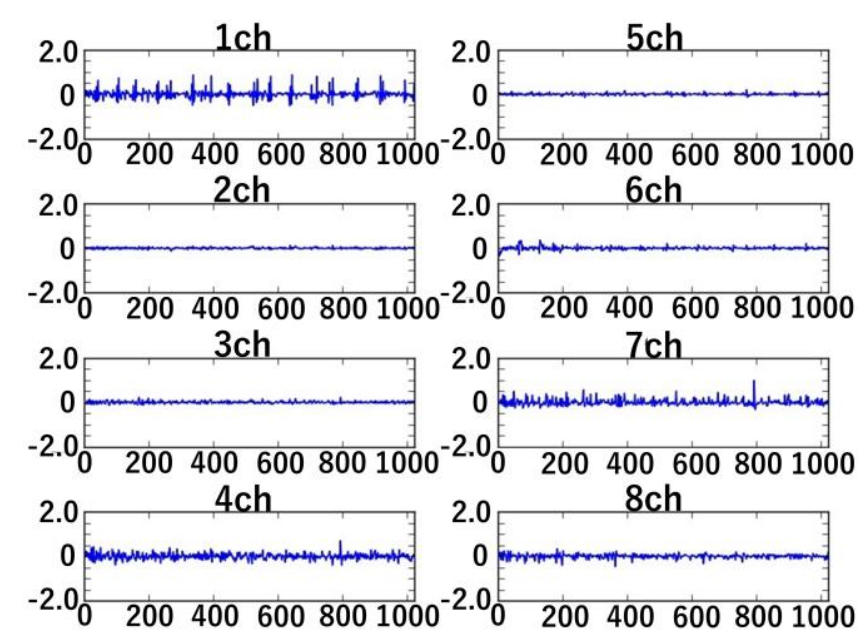

Fig. 9. Measurement Data after Preprocessing.

gathered 320 data in total. EMG data used for experiments is a part of each time series data in Fig.11. Fig.11 shows how to cut out the identification data. In addition, measurement data is divided into learning data and test data. We split 40 data of each subject in four. The 10 data is used as test data, the rest 30 data is as learning data for cross validation. It is possible to find out adversely affecting data in identification accuracy. Fig. 12 shows the combination for crossvalidation. However, learning data is few. Therefore, we subdivide each datum, which is composed of 1024 points. We divide a 1024 points datum into 8 parts, each of which has 128 points. As a result, we obtain eightfold increased data. Fig.13 shows how to split the data. After that, feature extraction and identification are performed using $\mathrm{CNN}$ and full connection layer.

\section{Result and consideration}

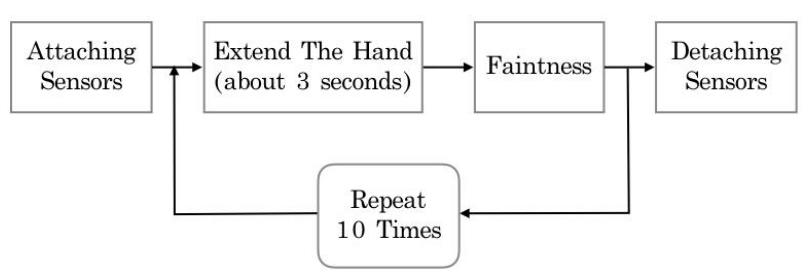

Fig. 10. Measurement Flow of EMG.

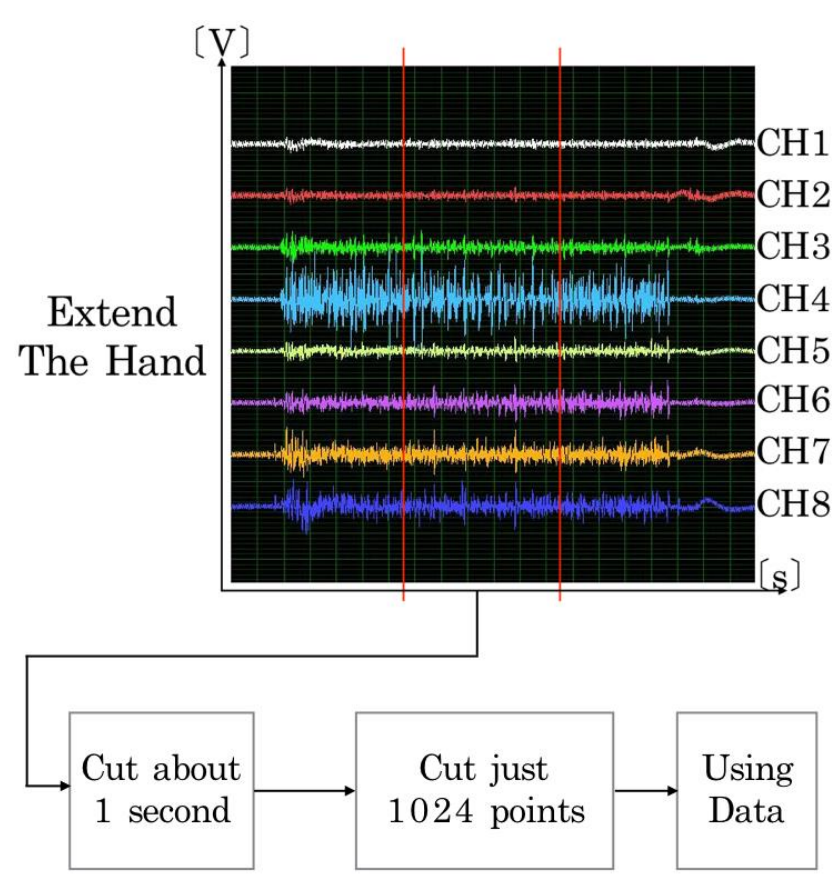

Fig. 11. How to Cut of the Data.

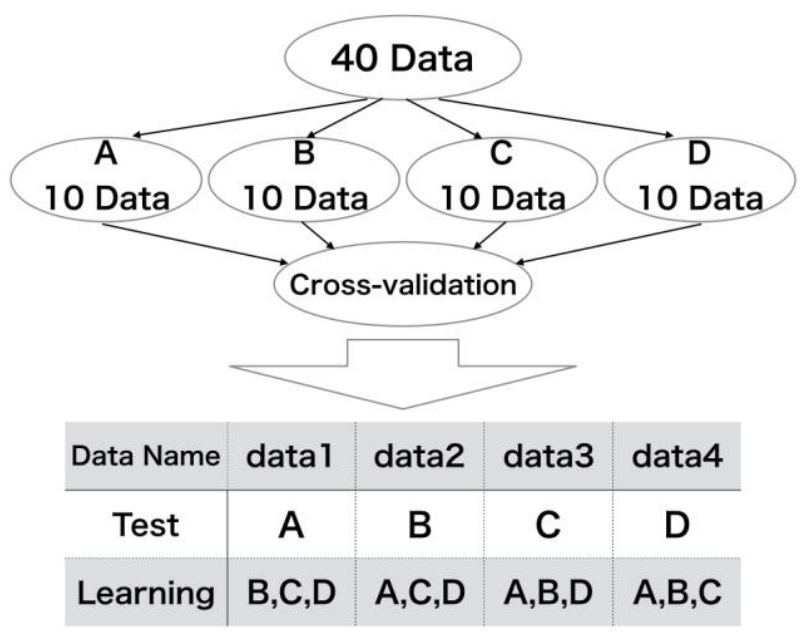

Fig. 12. Combination of data for crossvalidation.

Table 1 shows the experimental result of the conventional method and the proposed method. The true acceptance rate (TAR) increased by $7.2 \%$ and false acceptance rate (FAR) decreased by $0.0067 \%$. In addition, the identification accuracy increased by $1.5 \%$. Next, Tables 2 to 5 shows the change of true acceptance rate and false 
$8 \mathrm{CH}$

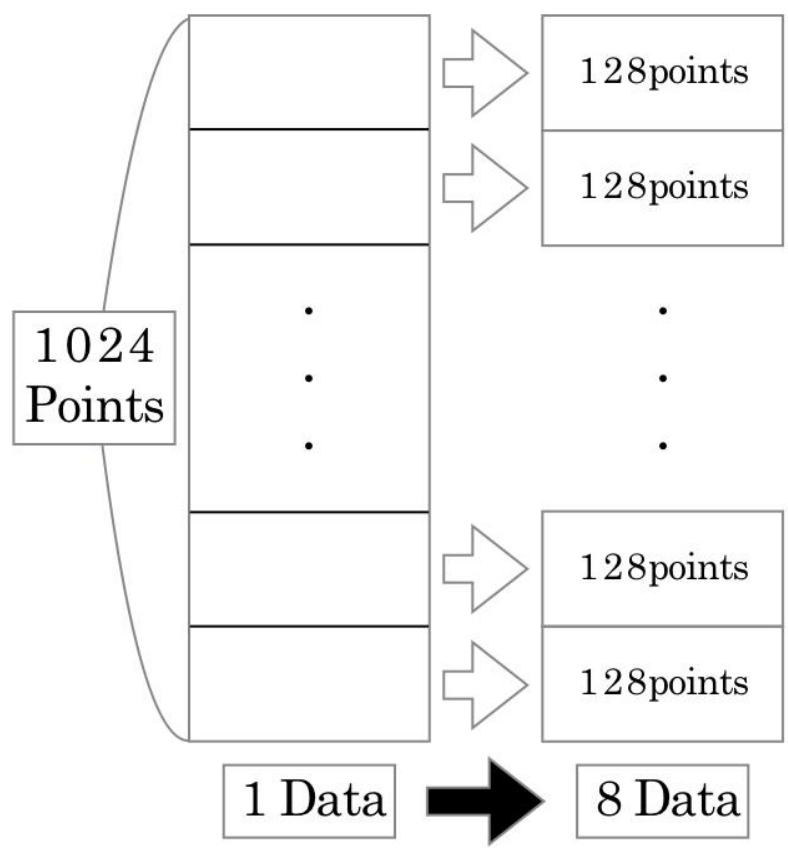

Fig. 13. How to split the data.

Table 1. Experimental Result.

\begin{tabular}{|c||c|c||c|}
\hline Perform HPF & TAR & FAR & Accuracy \\
\hline \hline No & $72.2 \%$ & $0.0157 \%$ & $95.2 \%$ \\
\hline Yes & $\mathbf{7 9 . 4 \%}$ & $\mathbf{0 . 0 0 9 0 \%}$ & $\mathbf{9 6 . 7 \%}$ \\
\hline
\end{tabular}

Table 2. True Acceptance Rate without Preprocessing.

\begin{tabular}{|c|c|c|c|c||c|}
\hline Subject & data1 & data2 & data3 & data4 & Average \\
\hline a & $40.0 \%$ & $95.0 \%$ & $99.0 \%$ & $26.5 \%$ & $\mathbf{6 5 . 1 \%}$ \\
\hline b & $39.3 \%$ & $100 \%$ & $86.8 \%$ & $0.0 \%$ & $\mathbf{5 6 . 5 \%}$ \\
\hline c & $94.3 \%$ & $76.3 \%$ & $69.8 \%$ & $93.8 \%$ & $\mathbf{8 3 . 5 \%}$ \\
\hline d & $54.5 \%$ & $96.0 \%$ & $44.3 \%$ & $70.0 \%$ & $\mathbf{6 6 . 2 \%}$ \\
\hline e & $91.5 \%$ & $71.8 \%$ & $9.5 \%$ & $45.5 \%$ & $\mathbf{5 4 . 6 \%}$ \\
\hline f & $62.0 \%$ & $87.0 \%$ & $60.5 \%$ & $81.3 \%$ & $\mathbf{7 2 . 7 \%}$ \\
\hline g & $100 \%$ & $79.0 \%$ & $99.8 \%$ & $98.0 \%$ & $\mathbf{9 4 . 2 \%}$ \\
\hline h & $64.3 \%$ & $84.0 \%$ & $100 \%$ & $92.5 \%$ & $\mathbf{8 5 . 2 \%}$ \\
\hline \hline Average & $\mathbf{6 8 . 2 \%}$ & $\mathbf{8 6 . 1 \%}$ & $\mathbf{7 1 . 2 \%}$ & $\mathbf{6 3 . 4 \%}$ & $\mathbf{7 2 . 2 \%}$ \\
\hline
\end{tabular}

acceptance rate by preprocessing. The average of false acceptance rate decreased for all combinations. The total average of true acceptance rate significantly increased. However, true acceptance rate of some subjects decreased. Fig.6 shows measurement data of the subject e, in which true acceptance rate significantly increased. In general, subjects with increased true acceptance rate contained a lot of drift noise in the measurement data. Therefore, it can be considered that the true acceptance rate was significantly
Table 3. True Acceptance Rate after Preprocessing.

\begin{tabular}{|c|c|c|c|c||c|}
\hline Subject & data1 & data2 & data3 & data4 & Average \\
\hline a & $13.8 \%$ & $99.0 \%$ & $96.5 \%$ & $47.5 \%$ & $\mathbf{6 4 . 2 \%}$ \\
\hline b & $54.0 \%$ & $100 \%$ & $97.3 \%$ & $0.0 \%$ & $\mathbf{6 2 . 8 \%}$ \\
\hline c & $100 \%$ & $67.0 \%$ & $89.3 \%$ & $93.3 \%$ & $\mathbf{8 7 . 4 \%}$ \\
\hline d & $84.8 \%$ & $97.5 \%$ & $42.8 \%$ & $83.8 \%$ & $\mathbf{7 7 . 2 \%}$ \\
\hline e & $97.0 \%$ & $97.8 \%$ & $32.3 \%$ & $95.0 \%$ & $\mathbf{8 0 . 5 \%}$ \\
\hline f & $96.3 \%$ & $93.5 \%$ & $68.3 \%$ & $83.5 \%$ & $\mathbf{8 5 . 4 \%}$ \\
\hline g & $99.1 \%$ & $95.5 \%$ & $100 \%$ & $96.5 \%$ & $\mathbf{9 3 . 4 \%}$ \\
\hline h & $55.3 \%$ & $81.8 \%$ & $100 \%$ & $99.8 \%$ & $\mathbf{8 4 . 2 \%}$ \\
\hline \hline Average & $\mathbf{7 4 . 8 \%}$ & $\mathbf{8 9 . 6 \%}$ & $\mathbf{7 8 . 3 \%}$ & $\mathbf{7 4 . 9 \%}$ & $\mathbf{7 9 . 4 \%}$ \\
\hline
\end{tabular}

Table 4. False Acceptance Rate without Preprocessing.

\begin{tabular}{|c|c|c|c|c||c|}
\hline Subject & data1 & data 2 & data3 & data4 & Average \\
\hline a & $0.0382 \%$ & $0.0 \%$ & $0.0032 \%$ & $0.0093 \%$ & $\mathbf{0 . 0 1 2 7 \%}$ \\
\hline b & $0.0029 \%$ & $0.0011 \%$ & $0.0089 \%$ & $0.0 \%$ & $\mathbf{0 . 0 0 3 2} \%$ \\
\hline c & $0.0075 \%$ & $0.0432 \%$ & $0.0261 \%$ & $0.0004 \%$ & $\mathbf{0 . 0 1 9 3 \%}$ \\
\hline d & $0.0411 \%$ & $0.0039 \%$ & $0.0346 \%$ & $0.0154 \%$ & $\mathbf{0 . 0 2 3 7 \%}$ \\
\hline e & $0.0314 \%$ & $0.0011 \%$ & $0.0014 \%$ & $0.0300 \%$ & $\mathbf{0 . 0 1 6 0 \%}$ \\
\hline f & $0.0039 \%$ & $0.0232 \%$ & $0.0168 \%$ & $0.0654 \%$ & $\mathbf{0 . 0 2 7 3 \%}$ \\
\hline g & $0.0 \%$ & $0.0504 \%$ & $0.0011 \%$ & $0.0021 \%$ & $\mathbf{0 . 0 1 3 4 \%}$ \\
\hline h & $0.0136 \%$ & $0.0154 \%$ & $0.0014 \%$ & $0.0096 \%$ & $\mathbf{0 . 0 1 0 0 \%}$ \\
\hline \hline Average & $\mathbf{0 . 0 1 7 3 \%}$ & $\mathbf{0 . 0 1 7 3 \%}$ & $\mathbf{0 . 0 1 1 7 \%}$ & $\mathbf{0 . 0 1 6 5 \%}$ & $\mathbf{0 . 0 1 5 7 \%}$ \\
\hline
\end{tabular}

Table 5. False Acceptance Rate after Preprocessing.

\begin{tabular}{|c|c|c|c|c||c|}
\hline Subject & data & data 2 & data 3 & data 4 & Average \\
\hline a & $0.0075 \%$ & $0.0 \%$ & $0.0046 \%$ & $0.0164 \%$ & $\mathbf{0 . 0 0 7 1 \%}$ \\
\hline b & $0.0004 \%$ & $0.0007 \%$ & $0.0018 \%$ & $0.0 \%$ & $\mathbf{0 . 0 0 0 7 \%}$ \\
\hline c & $0.0 \%$ & $0.0404 \%$ & $0.0100 \%$ & $0.0050 \%$ & $\mathbf{0 . 0 1 3 8 \%}$ \\
\hline d & $0.0250 \%$ & $0.0007 \%$ & $0.0257 \%$ & $0.0118 \%$ & $\mathbf{0 . 0 1 5 8 \%}$ \\
\hline e & $0.0064 \%$ & $0.0007 \%$ & $0.0 \%$ & $0.0161 \%$ & $\mathbf{0 . 0 0 5 8 \%}$ \\
\hline f & $0.0007 \%$ & $0.0086 \%$ & $0.0093 \%$ & $0.0496 \%$ & $\mathbf{0 . 0 1 7 1 \%}$ \\
\hline g & $0.0064 \%$ & $0.0236 \%$ & $0.0 \%$ & $0.0004 \%$ & $\mathbf{0 . 0 0 7 6 \%}$ \\
\hline h & $0.0086 \%$ & $0.0047 \%$ & $0.0025 \%$ & $0.0014 \%$ & $\mathbf{0 . 0 0 4 3 \%}$ \\
\hline \hline Average & $\mathbf{0 . 0 0 6 9 \%}$ & $\mathbf{0 . 0 0 9 9 \%}$ & $\mathbf{0 . 0 0 6 7 \%}$ & $\mathbf{0 . 0 1 2 6 \%}$ & $\mathbf{0 . 0 0 9 0 \%}$ \\
\hline
\end{tabular}

increased by the high path filter. On the other hand, Fig.15 shows the measurement data of the subject $a$, in which true acceptance rate decreased. In general, subjects with decreased true acceptance rate were not so much noisy from the beginning. Therefore, it can be considered that the characteristic of an individual has been reduced by performing a high path filter. Next, we focus on data with true acceptance rate of $0 \%$. The true acceptance rate of subject $\mathrm{b}$ is $0 \%$ in case of data4. Fig. 16 shows test data of data4. We can observe steady noise in $2 \mathrm{ch}$ and $3 \mathrm{ch}$. Fig. 17 

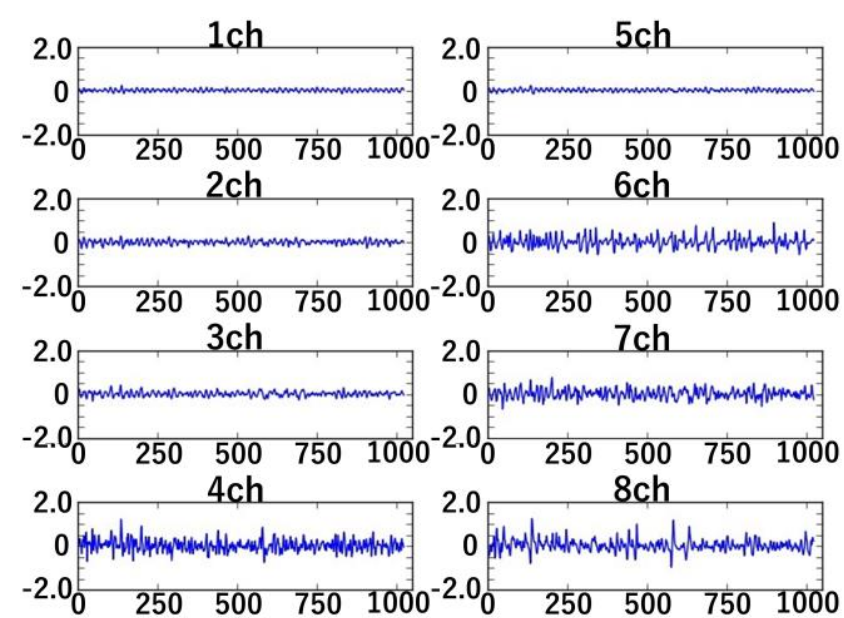

Fig. 15. The Measurement Data of the Subject a.
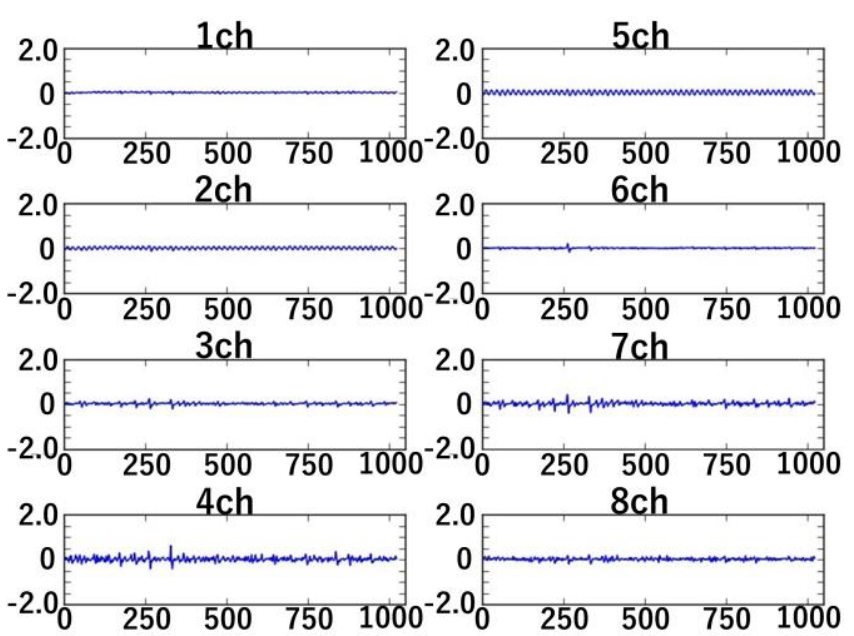

Fig. 16. Test data of Subject b in Data4.

shows the power spectra of test data. There is a peak that arises unnaturally and largely around $60 \mathrm{~Hz}$ of 2 ch and 3 ch. This noise was seen in all data on the same measurement date. Therefore, we conclude that misidentification can be caused by this noise. If this noise is eliminated, the true acceptance rate of data4 is considered to be increased. However, the individual processing for each data lacks versatility. On the other hand, if the $60 \mathrm{~Hz}$ band of all data is removed, the characteristics of other data can be diminished. In addition, it is considered that the whole accuracy is decreased. In future work, we should further analyze data and consider how to remove this noise.

\section{Conclusion}

In this paper we measured EMG by attaching dry type sensors to wrist, and carried out personal authentication. The proposed method was divided into three units such as a measuring, a feature extraction and discrimination units. We
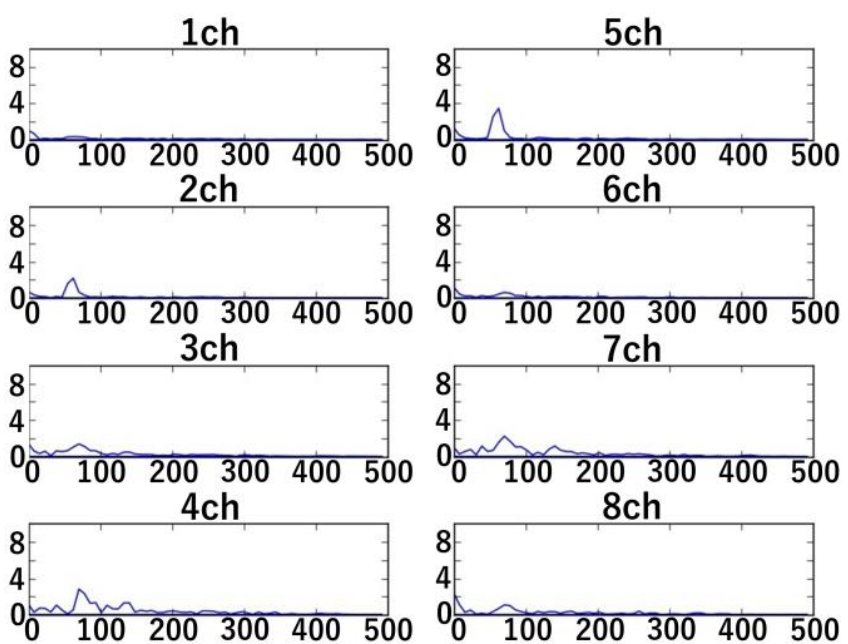

Fig. 17. Power Spectra of Test Data in Fig.16.

measured EMG signals with eight dry type sensors at the wrist. After that, we identified a motion opening our hand. We used a convolutional neural network (CNN) to learning and authentication. We collected 40 data for each subject. The average accuracy of two-class classification was $94.9 \%$ by CNN. In addition to the conventional method, the proposed method in this paper preprocessed EMG data. Large noises were removed using a high path filter. By this preprocessing, identification accuracy (Two-class classification using $\mathrm{CNN}$ ) was improved by $1.5 \%$. The true acceptance rate was improved by $7.2 \%$, and the false acceptance rate was improved by $0.0067 \%$. In future work, we review input data. We consider not only noise removal but also normalization to eliminate intra-individual difference. Furthermore, we review a layer structure of CNN.

This work was partly supported by JSPS KAKENHI Grant Number 16K01357.

\section{References}

(1) Osamu FUKUDA, Jun ARITA, and Toshiro TSUJI : "An EMG Controlled Omnidirectional Pointing Device", Trans. of Institute of Electronics, Information, and Communication Engineers, Vol. J87-D-2, No.10, pp.1996-2003, 2004

(2) Osamu Fukuda, Kouji Tsujimura, Toshio Tsuji and Akira Otsuka : "Control of a Meal-assistance Manipulator Using EMG Signals", Japanese Journal of Medical Instrumentation Vol.74, No.5, pp.229-237, 2004

(3) Daiki Hiraoka, Momoyo Ito, Shin-ichi Ito, Minoru Fukumi : "Japanese Janken Recognition by Support 
Vector Machine Based on Electromyogram of Wrist", Proc. of International Conference on Knowledge and Smart Technology, p.114-119, 2016

(4) Anita Pal, Ajeet Kumar Gautam, Yogendra Narain Slingh : "Evaluation of Bioelectric Signals for Human Recognition”, Procedia Computer Science 48(2015), p.746-752, 2015

(5) M. Abo-Zahhad, Sabah M.Ahmed, Sherif N.Abbas : "A New EEG Acquisition Protocol for Biometric Identification Using Eye Blinking Signals", Intelligent Systems and Applications, p.48-54, 2015.

(6) Noureddine Belgacem, Amine Nait-Ali, Regis Fournier, Fethi Bereksi-Reguig : "ECG BASED HUMAN AUTHENTICATION USING WAVELETS AND RANDOM FORESTS", International Journal on Cryptography and Information Security (IJCIS), Vol.2, No.2, 2012.

(7) B.Sabarigiri, D.Suganyadevi : "An Efficient Multimodal Biometric Authentication based on IRIS and Electroencephalogram (EEG) ", Proc. of Int. Conf. on Control, Communication and Power Engineering, CCPE, 2014.

(8) James Cannan, Huosheng $\mathrm{Hu}$ : "Authentication User Identification By Using Forearm Biometrics", International Conference on Advanced Intelligent Mechanics (AIM), 2013

(9) Ryohei Shioji, Daiki Hiraoka, Shin-ichi Ito, Momoyo Ito, Minoru Fukumi : "Personal Authentication Based on Wrist EMG Analysis by a convolutional Neural Network", Proc. of $4^{\text {th }}$ International Conference on Advanced Technology \& Sciences, p335-340 